\title{
THE NUMERICAL SIMULATION OF UNSTEADY CAVITATION EVOLUTION INDUCED BY PRESSURE WAVE*
}

\author{
B.C. KHOO \\ Department of Mechanical Engineering, National University of Singapore, \\ Singapore 119260, Singapore \\ Temasek Laboratories, National University of Singapore, \\ Singapore 117411, Singapore \\ mpekbc@nus.edu.sg \\ J.G. ZHENG \\ Temasek Laboratories, National University of Singapore, \\ Singapore 117411, Singapore \\ Department of Mechanical Engineering, National University of Singapore, \\ Singapore 119260, Singapore \\ tslzhen@nus.edu.sg
}

Published 17 November 2014

\begin{abstract}
The present study is focused on the numerical simulation of pressure wave propagation through the cavitating compressible liquid flow, its interaction with cavitation bubble and the resulting unsteady cavitation evolution. The compressibility effects of liquid water are taken into account and the cavitating flow is governed by one-fluid cavitation model which is based on the compressible Euler equations with the assumption that the cavitation is the homogeneous mixture of liquid and vapour which are locally under both kinetic and thermodynamic equilibrium. Several aspects of the method employed to solve the governing equations are outlined. The unsteady features of cavitating flow due to the external perturbation, such as the cavitation deformation and collapse and consequent pressure increase are resolved numerically and discussed in detail. It is observed that the cavitation bubble collapse is accompanied by the huge pressure surge of order of 100 bar, which is thought to be responsible for the material erosion, noise, vibration and loss of efficiency of operating underwater devices.
\end{abstract}

Keywords: Compressible flow; cavitation; pressure-wave-cavitation interaction.

\section{Introduction}

Cavitation is the formation and evolution of vapour bubble in a liquid flow and occurs when the pressure of liquid reaches saturation condition. The cavitation phenomenon is undesired in most situations and therefore preventing the cavitation occurrence has been the subject of a large body of research for the past several decades.

* This is an Open Access article published by World Scientific Publishing Company. It is distributed under the terms of the Creative Commons Attribution 3.0 (CC-BY) License. Further distribution of this work is permitted, provided the original work is properly cited. 
In the most of theoretical and numerical studies of cavitation, the flow is in low Mach number regime and therefore liquid is considered to be incompressible. This assumption is believed to be reasonable under a large number of situations and verified by the experimental observations ${ }^{4,10,11}$. One of the most popular approaches based on the incompressible treatment is the model of Kunz et $\mathrm{al}^{5}$. The method is seen to be able to accurately predict the transient and steady sheet and supercavitation flows. However, it is fairly complex and many parameters involved in the model have to be determined empirically. Another disadvantage of this type of method is that it cannot deal with the wave propagation and compressibility effects of liquid, which may be of great importance and must be taken into account in some engineering applications. An example is the cavitating object exposed to a nearby underwater explosion. Unfortunately, the available literature is limited on this subject ${ }^{12}$. Our study in this paper is focused on the unsteady dynamics of cavitation arising from the external pressure wave. Here, the viscous effects are considered to be less important and the cavitating flow is described by the time-dependent Euler equations. The phase transition is assumed to occur instantaneously and there are no empirical parameters in the model. The modified Tait equation of state (EOS) is employed to model liquid. The governing equations are solved using a second-order accurate, cell-centered finite volume method on structured, bodyfitted grid. The time-marching is handled using the explicit four-stage Runge-Kutta scheme.

The paper is organized as follows. In Section 2, the physical model and numerical method are described. In Section 3, the numerical results are presented and discussed. The conclusion is given in Section 4.

\section{Physical Model and Numerical Method}

\subsection{Governing equations}

The two-dimensional (2D) asymmetric Euler equations are written in conservative form as,

$$
\frac{\partial U}{\partial t}+\frac{\partial f}{\partial x}+\frac{\partial g}{\partial y}=-(i-1) H
$$

where $i$ takes on the value of 1 and 2 for planar and axisymmetric flows, respectively. Here, $U, f, g$ and $H$ are the vector of conserved variables, inviscid flux vectors in $x$ and $y$ directions, axisymmetric source terms, respectively, and given by,

$$
\begin{aligned}
& U=[\rho, \rho u, \rho v, \rho E]^{T} \\
& f=\left[\rho u, \rho u^{2}+p, \rho u v,(\rho E+p) u\right]^{T} \\
& g=\left[\rho v, \rho u v, \rho v^{2}+p,(\rho E+p) v\right]^{T} . \\
& H=1 / y\left[\rho v, \rho u v, \rho v^{2},(\rho E+p) v\right]^{T}
\end{aligned}
$$


with

$$
\begin{array}{ll}
\rho=\alpha_{v} \rho_{v, \text { sat }}+\left(1-\alpha_{v}\right) \rho_{l, s a t,} & E=e+\left(u^{2}+v^{2}\right) / 2 \\
\rho e=\alpha_{v} \rho_{v, \text { sat }} e_{v, \text { sat }}+\left(1-\alpha_{v}\right) \rho_{l, \text { sat }} e_{l, \text { sat },} & \alpha_{v} \in[0,1]
\end{array} .
$$

In the equations above, $\rho, p, E u, v$ are the mixture density, pressure, total energy, $x$ and $y$ velocity components, respectively. In addition, $\alpha_{v}, \rho_{v, s a t}$ and $e_{v, s a t}$ denote the volume fraction of vapour, saturated vapour density and energy. The quantities with subscript $l$ correspond to the saturation condition of liquid water.

\subsection{Equations of state}

To close system (1), equations of state have to be provided, which are suitable to describe the properties of liquid water, water vapour and saturated water-vapour mixture.

For liquid phase, an established EOS is the modified Tait $\operatorname{EOS}^{4,8,13}$ and given by,

$$
p=p(\rho, T)=B\left[\left(\frac{\rho}{\rho_{l, s a t}(T)}\right)^{N}-1\right]+p_{\text {sat }}(T),
$$

where $B$ and $N$ are the material-dependent constants and take the values of $3.3 \times 10^{8} \mathrm{~Pa}$ and 7.5, respectively for liquid water. $\rho_{l, \text { sat }}$ and $p_{\text {sat }}$ are the saturated liquid density and pressure at temperature $T$. A thermally consistent description for the associated internal energy takes the form,

$$
e_{l}(T)=C_{v l}\left(T-T_{r e f}\right)+e_{l r e f}
$$

where $C_{v l}=4186 \mathrm{~J} / \mathrm{kg} \cdot \mathrm{K}$ is the specific heat of water at constant volume, $T_{\text {ref }}=273.15 \mathrm{~K}$ is the reference temperature and $e_{\text {lref }}=617 \mathrm{~J} / \mathrm{kg}$ is the reference internal energy at the reference temperature $T_{\text {ref }}$.

For the pure water vapour, the ideal EOS is employed,

$$
p=\rho R T
$$

where $\mathrm{R}=461.5 \mathrm{~J} / \mathrm{kg} \cdot K$ is the gas constant. The corresponding caloric EOS is given by,

$$
e_{v}(T)=C_{v v}\left(T-T_{r e f}\right)+L_{v r e f}+e_{l r e f}
$$

with $C_{v v}=1410.8 \mathrm{~J} / \mathrm{kg} \cdot \mathrm{K}$ is the specific heat of vapour at constant volume and $L_{v r e f}$ is the latent heat of vaporization and takes the value of $2.5013 \times 10^{6} \mathrm{~J} / \mathrm{kg} \cdot K . T_{\text {ref }}$ and $e_{\text {lref }}$ are defined in Eq. (5).

In the cavitation region, the vapour and liquid are assumed to be in the thermodynamic equilibrium, i.e. $T_{v}=T_{l}=T_{\text {sat }}$ and $p_{v}=p_{l}=p_{\text {sat }}$. The saturated pressure, liquid and vapour densities are calculated using the well-know Oldenbourg polynomials $^{4,8,13}$, 


$$
\begin{aligned}
& \ln \left(p_{s a t} / p_{c r}\right)=T_{c r} / T_{s a t}\left(a_{1} \theta+a_{2} \theta^{1.5}+a_{3} \theta^{3}+a_{4} \theta^{3.5}+a_{5} \theta^{4}+a_{6} \theta^{7.5}\right) \\
& \rho_{l, s a t} / \rho_{c r}=1+b_{1} \theta^{\frac{1}{3}}+b_{2} \theta^{\frac{2}{3}}+b_{3} \theta^{\frac{5}{3}}+b_{4} \theta^{\frac{16}{3}}+b_{5} \theta^{\frac{43}{3}}+b_{6} \theta^{\frac{110}{3}} \\
& \ln \left(\rho_{v, s a t} / \rho_{c r}\right)=c_{1} \theta^{\frac{2}{6}}+c_{2} \theta^{\frac{4}{6}}+c_{3} \theta^{\frac{8}{6}}+c_{4} \theta^{\frac{18}{6}}+c_{5} \theta^{\frac{37}{6}}+c_{6} \theta^{\frac{71}{6}}
\end{aligned}
$$

where $\theta=1-T / T_{c r}$ and the critical conditions for water are $T_{c r}=647.096 K, p_{c r}=2.2064 \times$ $10^{7} \mathrm{~Pa}$ and $\rho_{c r}=322 \mathrm{~kg} / \mathrm{m}^{3}$. The parameters in Eq. (8) are listed in Table 1.

Table 1. Oldenbourg polynomials coefficients.

\begin{tabular}{llll}
\hline Index & $\mathrm{a}$ & $\mathrm{b}$ & $\mathrm{c}$ \\
\hline 1 & -7.85823 & 1.99206 & -2.02957 \\
2 & 1.83991 & 1.10123 & -2.68781 \\
3 & -11.7811 & -0.512506 & -5.38107 \\
4 & 22.6705 & -1.75263 & -17.3151 \\
5 & -15.9393 & -45.4485 & -44.6384 \\
6 & 1.77516 & $-6.75615 \times 10^{5}$ & -64.3486 \\
\hline
\end{tabular}

The temperature is determined from the definition of mixture internal energy given in Eq. (3),

$$
e(T)=\left\{\left[\alpha_{v} \rho_{v, s a t}(T) C_{v v}+\left(1-\alpha_{v}\right) \rho_{l, s a t}(T) C_{v l}\right]\left(T-T_{r e f}\right)+\alpha_{v} \rho_{v, s a t}(T) L_{v r e f}\right\} / \rho+e_{\text {lref }}
$$

The sound speed of the mixture can be derived theoretically and given by ${ }^{1}$,

$$
a=\left\{\rho\left[\frac{\alpha_{v}}{\rho_{v, s a t} a_{v}^{2}}+\frac{1-\alpha_{v}}{\rho_{l, s a t} a_{l}^{2}}\right]\right\}^{-\frac{1}{2}}
$$

where $a_{v}$ and $a_{l}$ are the speeds of sound of vapour and liquid at the saturated temperature, respectively and given as,

$$
a_{v}=\sqrt{\gamma R T}, \quad a_{l}=\sqrt{N(p+B) / \rho}
$$

with $\gamma=1.327$.

\subsection{Numerical algorithm}

In this subsection, the main numerical algorithms of our computational fluid dynamics (CFD) code are described briefly. The method of lines allows the spatial and temporal derivatives to be discretized separately and offers us a lot of flexibility to select the appropriate numerical schemes for the spatial and temporal discretizations, separately. 
The system of compressible Euler equations (1) is solved using a cell-centered finite volume method. The computational domain is divided into a finite number of quadrilateral cells. The body-fitted mesh is structured and each cell is uniquely identified by the indices $(i, j)$ as shown in the schematic cell of Fig. 1. The governing equations (1) are integrated in each cell and the semi-discrete integral form corresponding to control volume $(i, j)$ in Fig. 1 is written as,

$$
\frac{\partial}{\partial t}\left(U_{i, j} \Omega_{i, j}\right)=-\sum_{m=1}^{4} \tilde{F}_{m} \cdot S_{m}-H_{i, j} \Omega_{i, j}=R_{i, j}(U)
$$

where the vector of conserved variables $U_{i, j}$ is associated with the cell centroid. $\tilde{F}_{m}$ represents the numerical approximation of convective fluxes across the control volume face with side vector $S_{m}$ and $\Omega_{i, j}$ is the area of cell. The numerical flux is calculated using the classical Harten-Lax-van Leer-Contact (HLLC) approximate Riemann solver ${ }^{3}$. The left and right input states with respect to the cell face is reconstructed with the second-order monotone upstream-centered schemes for conservation laws (MUSCL) ${ }^{2,} 9$ enhanced by an appropriate limiter.

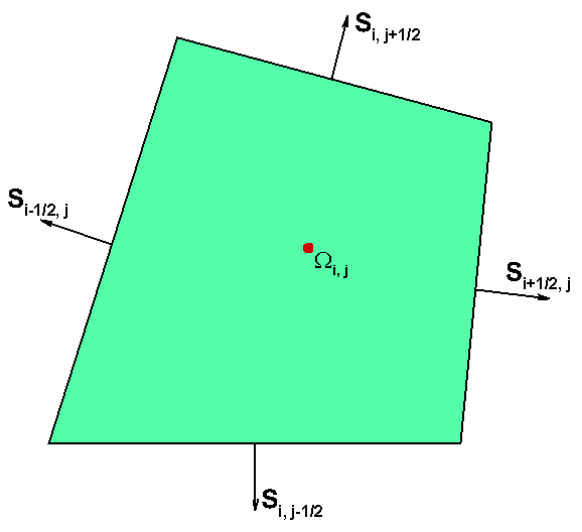

Fig. 1. Finite control volume $\Omega_{\mathrm{i}, \mathrm{j}}$ and normal vectors.

The time-marching is handled using the explicit, four-stage Runge-Kutta scheme by Jameson $^{6}$,

$$
\begin{aligned}
& U_{i, j}^{(0)}=U_{i, j}^{n} \\
& U_{i, j}^{(1)}=U_{i, j}^{(0)}-\alpha_{1} R_{i, j}^{(0)} \\
& U_{i, j}^{(2)}=U_{i, j}^{(0)}-\alpha_{2} R_{i, j}^{(1)} \\
& U_{i, j}^{(3)}=U_{i, j}^{(0)}-\alpha_{3} R_{i, j}^{(2)} \\
& U_{i, j}^{n+1}=U_{i, j}^{(4)}=U_{i, j}^{(0)}-\alpha_{4} R_{i, j}^{(3)}
\end{aligned}
$$


with $\Delta$ t being time step constrained by Courant-Friedrichs-Lewy (CFL) condition. The stage coefficients are optimized to enlarge stability region and take the values of $\alpha_{1}=$ $0.1084, \alpha_{2}=0.2602, \alpha_{3}=0.5052$ and $\alpha_{4}=1.0$.

\section{Numerical Results}

The one- and two-dimensional cavitating flows are resolved. The cavitation number which represents the surrounding flow conditions is defined as $\sigma=0.5\left(p_{\infty}-p_{\text {sat }}\right) /\left(\rho_{\infty} U_{\infty}^{2}\right)$ with $p_{\infty}, \rho_{\infty}, U_{\infty}$ being the freestream pressure, density and velocity, respectively and $p_{\text {sat }}$ being the saturated pressure. In all the calculations below, the CFL number is set to be 0.92 .

\subsection{One-dimensional shock tube problems}

The first case is the classical one-dimensional single phase Riemann problem. The $1 \mathrm{~m}$ long shock tube is filled with water at the temperature of $T=293 \mathrm{~K}$. Water in the region to the left of $x=0.5 \mathrm{~m}$ is highly pressurized with the pressure of $p_{L}=2.5 \times 10^{8} \mathrm{~Pa}$, whereas the right side is the low pressure region with $p_{R}=2500 P a$. Fluid is at rest initially.

Fig. 2 depicts the distributions of density, pressure, velocity and void fraction at time $\mathrm{t}=0.2 \mathrm{~ms}$. The governing equations (1) are solved on a uniform mesh of 500 cells in $x$ direction. The solution consists of a leftward-going rarefaction, a rightward-going shock wave and a contact discontinuity in between. Our code is seen to accurately capture all these flow structures without the presence of unphysical oscillations across the discontinuities.
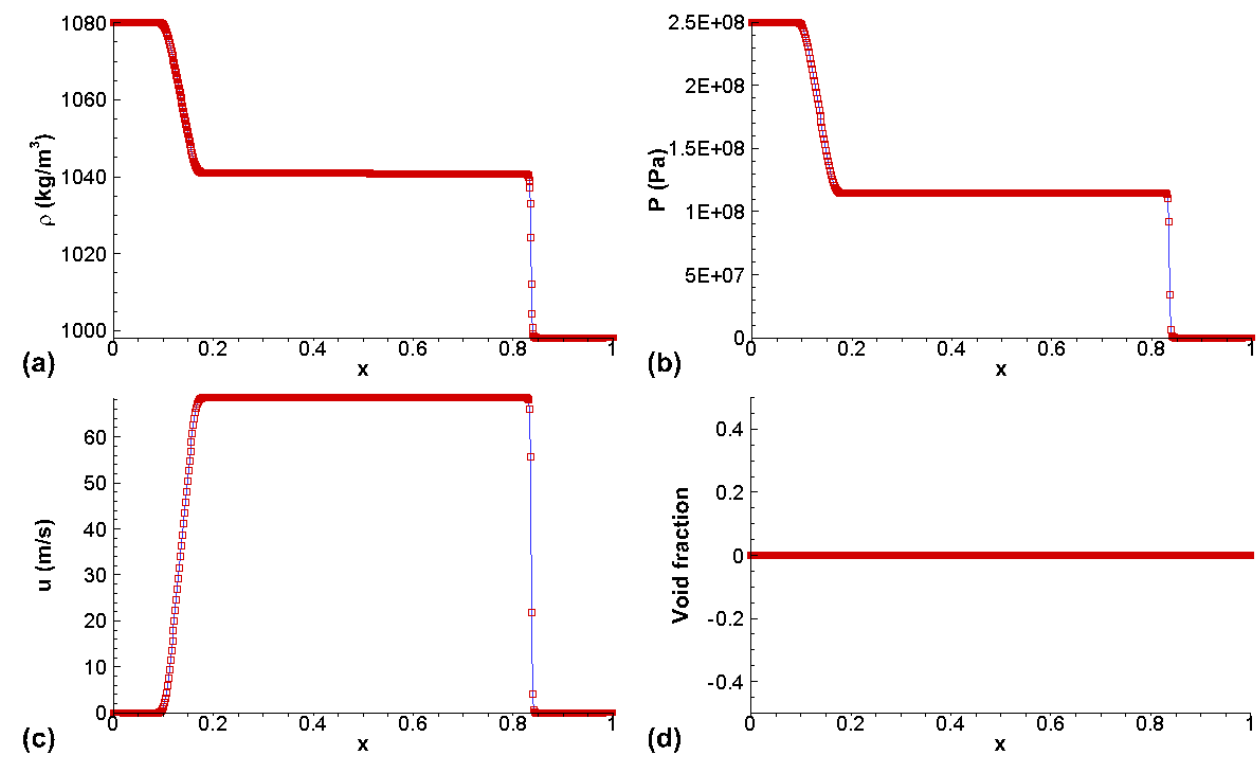

Fig. 2. The solution of one-dimensional Riemann problem at $\mathrm{t}=0.2 \mathrm{~ms}$. 
Next, the one-dimensional cavitation is simulated ${ }^{7}$. Initially, two water streams meet at the center of the $1 \mathrm{~m}$ long tube under the uniform pressure of $10^{8} \mathrm{~Pa}$ and temperature of $293 K$. The two streams are moving in the opposite directions with the same velocity magnitude of $100 \mathrm{~m} / \mathrm{s}$.

The initial condition will give rise to a left rarefaction and a right rarefaction. The liquid water around the tube center is to be vapourized, forming a cavitation bubble. The flow features are all well captured as illustrated in Fig. 3. The pressure decreases across the rarefaction waves and reaches the saturation value, which is around 5 orders of magnitude smaller than the initial data. The physically reasonable results are obtained with our code. The two simulations demonstrate the potential of our code to predict the wave dynamics.

(a)
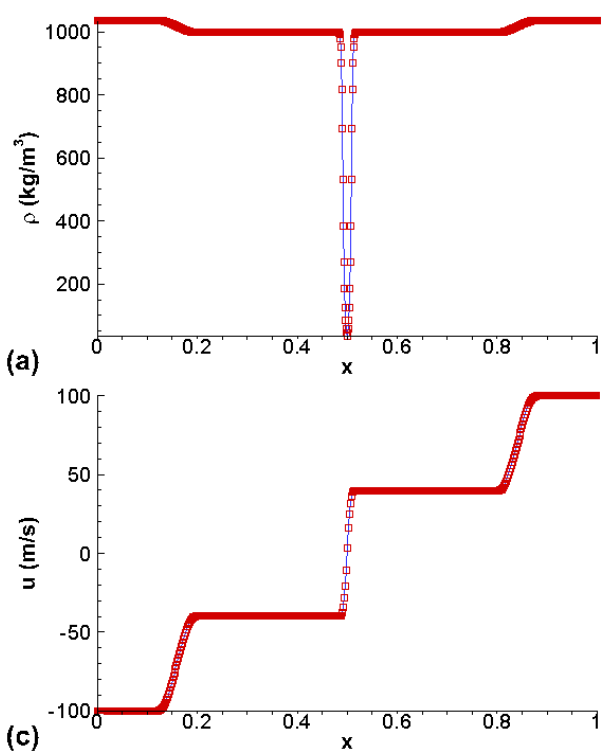

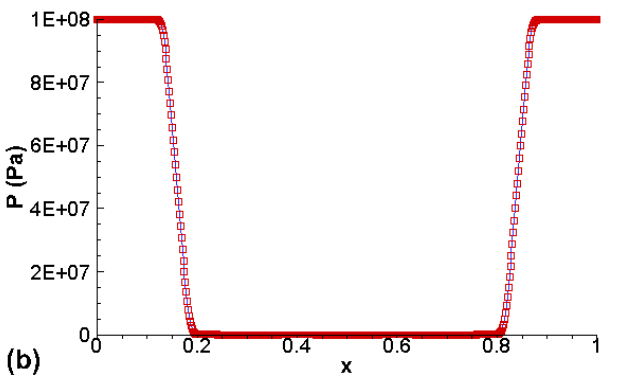

(d)

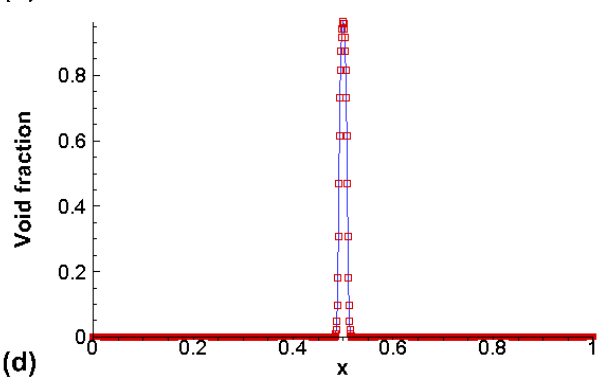

Fig. 3. The solution of one-dimensional cavitating flow at $\mathrm{t}=0.2 \mathrm{~ms}$.

\subsection{Two-dimensional partial cavitation}

The axisymmetric cavitating water flow around a cylinder of radius of $10 \mathrm{~mm}$ at zero angle of attack is resolved. The computational domain is large enough to minimize the influence of boundary condition implementation on the flow filed calculation. A mesh of $360 \times 80$ cells is used in the calculation on the upper half of flow field. At the inlet and outlet, the characteristic-based boundary conditions are imposed with a background pressure specified at the outlet. For the current partial cavitation simulation, the freestream flow speed of $U_{\infty}=25 \mathrm{~m} / \mathrm{s}$ and pressure of $p_{\infty}=10^{5} \mathrm{~Pa}$ results in a cavitation number of 0.3 at $T_{\infty}=293 \mathrm{~K}$. 
The transient flow fields from our simulation are shown in Fig. 4 where the left column corresponds to the density filed whereas the right column demonstrates the volume fraction contours with selected streamlines. After the cavitation bubble reaches its maximum size as shown in Fig. 4 (a1)-(b1), the trailing edge of cavity is characterized by an unsteady re-entrant jet. The significant flow recirculation is observed in Fig. 4 (a2)(b2). The re-entrant jet moves upstream along the cylinder surface, interacting with the leading edge of cavity, pinching off the bubble and leading to cavitation shedding, see the last two rows of Fig. 4. Our results qualitatively agree well with those reported in Refs. 5 and 14.
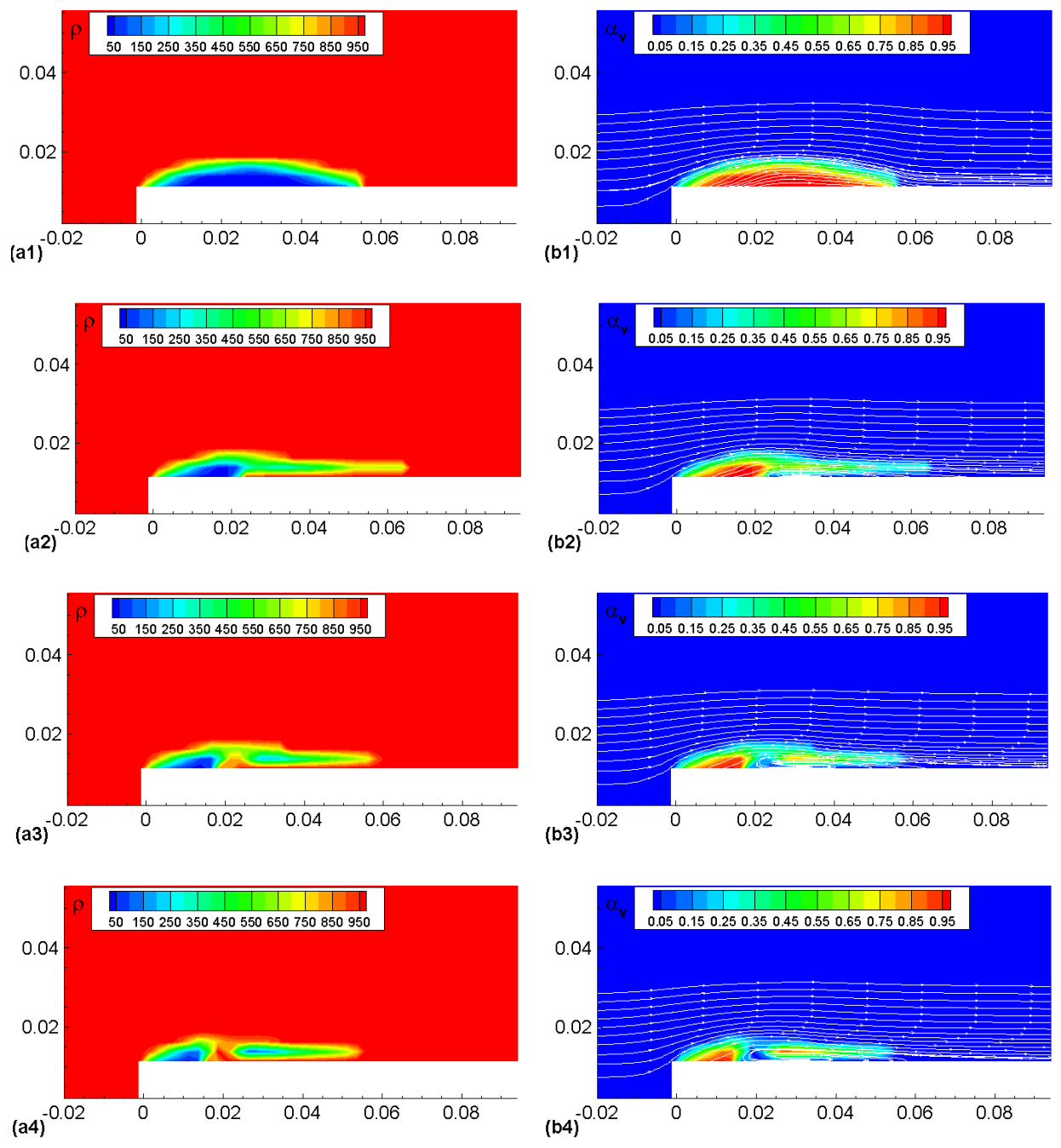

Fig. 4. The time evolution of a partial cavitation, $\sigma=0.3$. 


\subsection{Two-dimensional unsteady super-cavitation}

With increasing the freestream speed, the generated cavity will be longer and envelop the object, forming supercavity. For the super-cavitation calculation, the freestream velocity is $U_{\infty}=500 \mathrm{~m} / \mathrm{s}$, which corresponds to the cavitation number of $\sigma=1.95 \times 10^{-4}$. The unsteady simulation is based on the converged solution of super-cavitation flow with the above initial condition. In the next step, the freestream velocity is increased suddenly to generate a pressure wave at inlet which is to move downstream and impact on the supercavity.
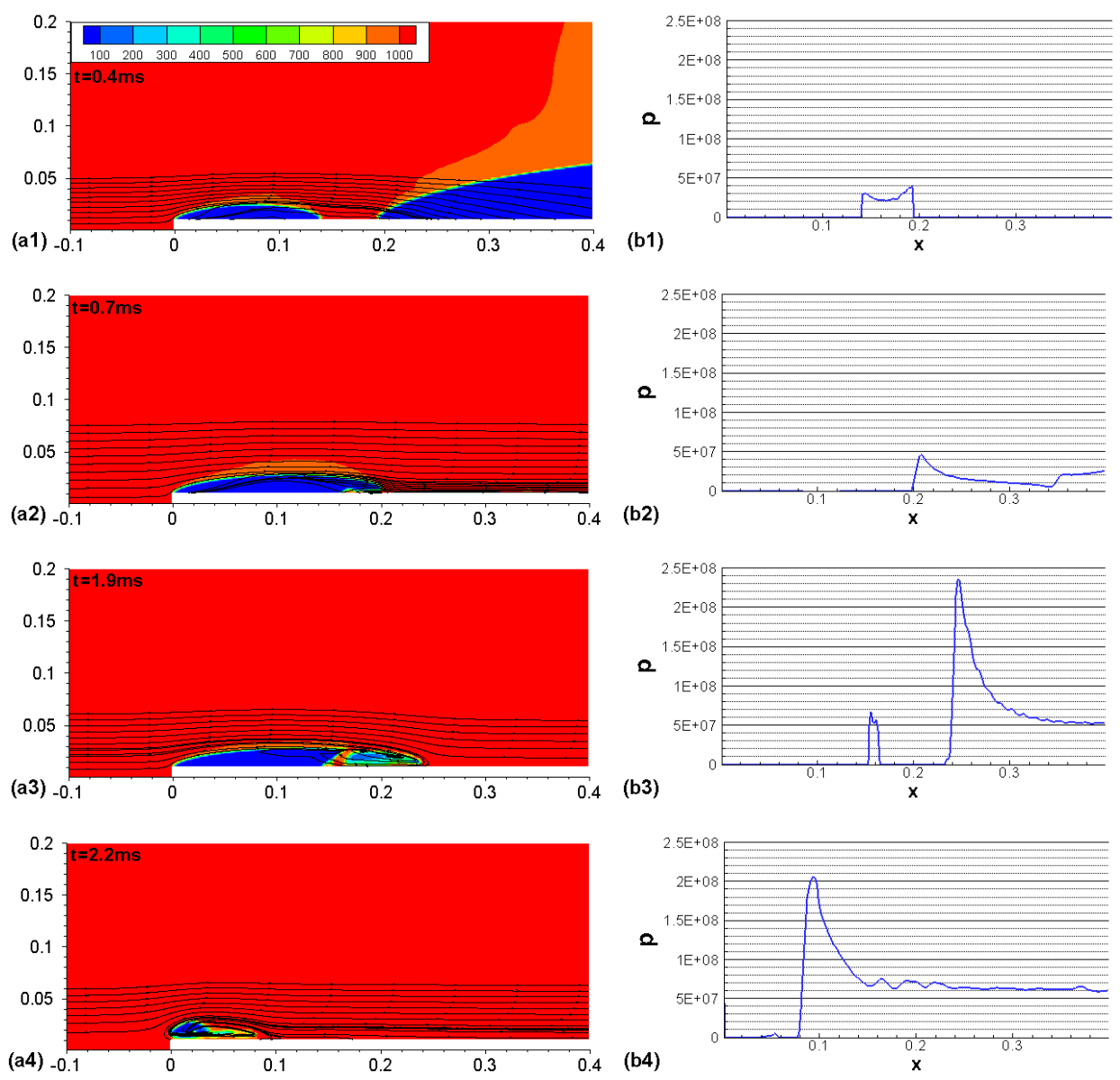

Fig. 5. The time evolution of super-cavitation impacted by a pressure wave.

The time sequence of predicted density field with the corresponding pressure distribution along cylinder surface is presented in Fig. 5 for a sudden freestream velocity increase of $\Delta U_{\infty}=50 \mathrm{~m} / \mathrm{s}$. The supercavity has been split into two parts by the pressure 
wave $0.4 m s$ after the freestream velocity is increased, as shown in Fig. 5 (a1). Two pressure surges are present in the pressure profile depicted in Fig. 5 (b1). One is immediately behind the closure region of the upstream cavity and the other is immediately before the leading edge of the downstream cavity. This results from the local collapse of cavity. The pressure increase associated with the left cavity collapse is high enough to create an adverse pressure gradient at the trailing edge. This leads to the flow recirculation and re-entrant jet, see the streamlines in Fig. 5 (a2). In Fig. 5 (a3) at $\mathrm{t}=1.9 \mathrm{~ms}$, the re-entrant jet moves further upstream and causes the cavitation shedding. The jet flow has reached the cylinder head at $\mathrm{t}=2.2 \mathrm{~ms}$ and eventually leads to the full collapse of cavity. The right cavity continues shrinking from its leading edge and vanishes quickly.
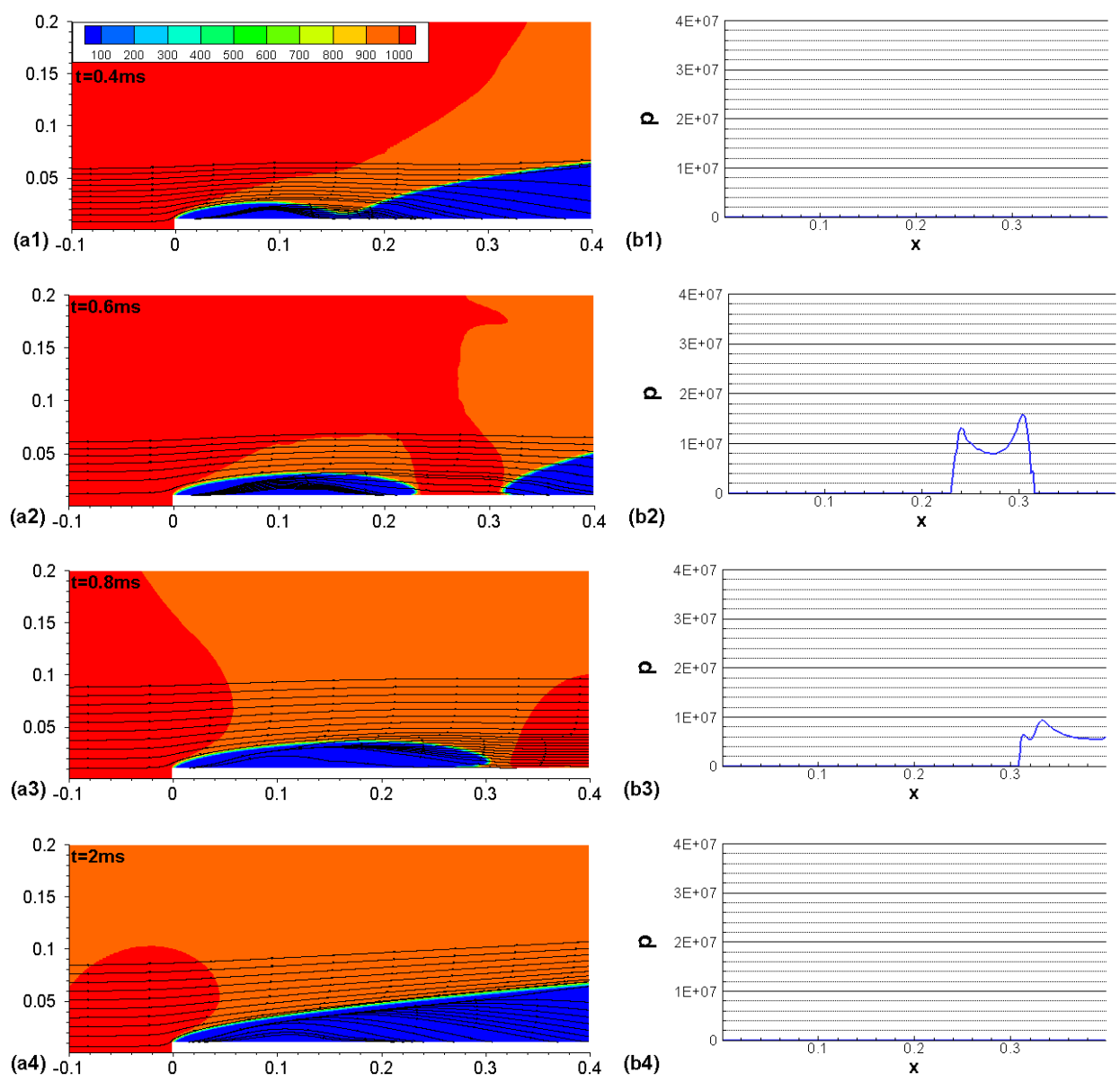

Fig. 6. The time evolution of super-cavitation impacted by a weak pressure wave. 
The time evolution of flow filed with $\Delta U_{\infty}=30 \mathrm{~m} / \mathrm{s}$ is shown in Fig. 6. For this case, the generated pressure wave at inlet is weaker. The left partial cavity breakup is accompanied by a weaker pressure surge. As a result, there is no re-entrant jet formed and the left cavity expands downstream, developing into a new supercavity.

\section{Conclusions}

The unsteady cavitating flow arising from the pressure wave impact has been numerically resolved using an in-house developed CFD code based on the homogeneous cavitation model. It is found that the re-entrant jet is responsible for the complete collapse of the upstream cavity. However, if the introduced pressure wave is not relatively high, the partial cavity can grow into a new supercavity.

\section{Acknowledgments}

The work is supported by ONR (Office of Naval Research) under grant number N000141010474.

\section{References}

1. G. B. Wallis, One-dimensional two-phase flow, (New York: McGill-Hill, 1969).

2. B. van Leer, Towards the ultimate conservative difference scheme $V$. A second-order sequel to Godunov's method, J. Comput. Phys. 32, 101 (1979).

3. E. F. Toro, Riemann solvers and numerical methods for fluid Dynamics: a practical introduction, (Springer-Verlag, Berlin Heidelberg, 1999).

4. R. Saurel, J.P. Cocchi and P.B. Butler, Numerical study of cavitation in the wake of a hypervelocity underwater projectile, J. Propul. Power, 15(4), 513 (1999).

5. R. F. Kunz, D. A. Boger and D. R. Stinebring, A preconditioned Navier-Stokes method for two-phase flows with application to cavitation prediction, Comput. Fluids, 29, 849 (2000).

6. J. Blazek, Computational fluid dynamics: principles and applications, (ELSEVIER, 2001).

7. T. G. Liu, B. C. Khoo and W. F. Xie, Isentropic one-fluid modeling of unsteady cavitating flow, J. Comput. Phys., 201, 80 (2004).

8. M. D. Neaves and J. R. Edwards, All-speed time-accurate underwater projectile calculations using a preconditioning algorithm, J. Fluids Eng., 128, 284 (2006).

9. B. van Leer, Upwind and High-Resolution Methods for Compressible Flow: From Donor Cell to Residual-Distribution Schemes, Commun. Comput. Phys., 1(2), 192 (2006).

10. S. J. Schmidt, I. H. Sezal, G. H. Schnerr and M. Talhamer, Riemann techniques for the simulation of compressible liquid flows with phase-transition at all Mach numbers - shock and wave dynamics in cavitating 3-D micro and macro systems, AIAA paper, 092407, 1 (2008).

11. S. J. Schmidt, M. Talhamer and G. H. Schnerr, Inertia controlled instability and small scale structures of sheet and cloud cavitation, Proceedings of the $7^{\text {th }}$ International Symposium on Cavitation, 17, 1 (2009).

12. J. G. Zheng, B. C. Khoo and Z. M. Hu, Simulation of wave-flow-cavitation interaction using a compressible homogenous flow method, Commun. Comput. Phys., 14(2), 328 (2012).

13. D. M. Causon and C. G. Mingham, Finite volume simulation of unsteady shock-cavitation in compressible water, Int. J. Numer. Meth. Fluids, DOI: 10.1002/d.3754, (2012).

14. L. X. Zhang and B. C. Khoo, Computations of partial and super cavitating flows using implicit pressure-based algorithm (IPA), Comput. Fluids, 73, 1 (2013). 